\title{
TRANSIENT ANALYSIS AND OPTIMIZATION OF ANNULAR ELLIPTICAL FINS WITH MIXED BOUNDARY CONDITION
}

\section{NAGENDRABABU MAHAPATRUNI ${ }^{1}$, SWATHI BOOSALA ${ }^{2} \&$ SRIKANTH SATISH KUMAR DARAPU ${ }^{3}$}

${ }^{1}$ AssociateProfessor, Department of Mechanical Engineering, Vignan's Institute of Engineering for Women, Visakhapatnam, India

${ }^{2}$ Assistant. Professor, Department of Mechanical Engineering, Vignan's Institute of Engineering for Women, Visakhapatnam, India

${ }^{3}$ Sr. Assistant Professor, Department of Civil Engineering, GIT, Gandhi Institute of Technology and Management (GITAM Deemed to be University), Visakhapatnam, India

\begin{abstract}
Fins increase the rate of heat dissipation to surrounding atmosphere. Different geometries of the fins show different heat transfer rate. This paper gives comparative heat transfer synopsis between circular annular fins and elliptical annular fins in transient state. Heat dissipation for elliptical fins with different aspect ratios have been modelled and simulated. Energy balance for the fin is done taking into consideration all three modes i.e., conduction, convection and radiation. Transient analysis for $200 \mathrm{sec}$ is done for both circular and elliptical annular fins. Results show that elliptical fins give high temperature gradient with time when compared to former. As the aspect ratio of the elliptical fins increases the slope of temperature gradient with respect to dimensionless time i.e., Fourier number goes on decreasing. The temperature gradient for elliptical annular fin with aspect ratio 0.1 and a circular annular fin whose aspect ratio is 1 are observed to be same during mathematical modelling. Effectiveness of elliptical annular fins with varying aspect ratio was plotted with Fourier number. When compared to circular annular fins later one show high effectiveness enhancement with decrease in aspect ratio. Efficiency of the fins with different aspect ratio were compared and observed that the maximum efficiency value for lower aspect ratios is high.
\end{abstract}

KEYWORDS: Elliptical fin, Numerical modelling, Simulation, Temperature gradient \& Aspect ratio

Received: Apr 07, 2020; Accepted: Apr 27, 2020; Published: Jul 11, 2020; Paper Id.: IJMPERDJUN2020283

\section{INTRODUCTION}

Fins are used to dissipate heat to surroundings. There are much geometry of fins available for effective transfer of heat from the base of the component. In this regard circular annular fins are used to dissipate heat from the heat exchanger body i.e., a long pipe. But same amount of heat dissipation can be achieved by elliptical annular fins. The material required for manufacturing of elliptical annular fin with its major axis equal to diameter of a circular annular fin is less for an aspect ratio of 0.9. As the aspect ratio of the elliptical fin is reduced the temperature gradient with respect to time has been increased. It results that same amount of temperature difference can be achieved with in less time when compared to circular annular fins. The space occupied by circular fins is more when compared to elliptical fins. Elliptical fins are used in some rocket application as they produce least amount of drag when compared to other geometries of fins such as trapezoidal, square, rectangular, circular and clipped data. Elliptical fins can be attached to the wall of the base component strongly due to their right direction of orientation of the 
grains. Hisham H. Jasim and Mehmet Sait Soylemezgave experimental results for a perforated fin with different inclination angles. In order to maximize heat transfer rate surface of the fins are provided with perforations. Rectangular pin fins were provided with elliptical perforation in order improve heat dissipation from the fin. It was observed that use of perforations decrease the thermal resistance of the fin and improves its thermal performance. Due to perforations and inclinations the rate of heat transfer is increased as the Nusselt number of the fin is also increased hence convective heat transfer coefficient [4]. As the number of fins and perforations increase the weight of the fin material and its volume gets reduced hence increasing Peclet number in forced convection and Rayleigh number free convection [4]. This type of alignment also increases the strength of the fin as well as strength of the component. As compared to rest of the geometries elliptical fins require less material for manufacturing and they are light weight. Elliptical fins are used for stabilization of the component by folding them over the periphery of the component. N.Nagarani and K.Mayilasamy demonstrated about performance of elliptical annular fin in steady state and with natural convection as well as forced convection. Efficiency of elliptical annular fins was compared with circular annular fins in different environment conditions. They found that if space is restricted in one direction then efficiency of elliptical fin is greater than that of circular fin [1].For effective working of elliptical fin it is optimized with effectiveness of the fin as objective function and constant volume and thickness of the fin as constraints using genetic algorithm by N. Nagarani et al. It was observed that effective ness of the fin is maximum when minor axis of the elliptical fin touches the circumference of the circular tube and when the shape factor of the fin is also low [2]. Optimization of fin geometry is given by H. Nemati and S. Samivand. As the aspect ratio of the fin increases the circular shape of temperature lines doesn't remain instead they start to deviate from the centre of the fin they show parabolic nature. Hence for evaluating fin efficiency fin effective area method and sector method can't be used. In order to overcome this, a correlation using polar coordinate system was derived and geometry of the fin was optimized. Through this method rate of heat dissipation for specified volume of the fin was maximized [3].OguzUzol gave experimental analysis of elliptical fin compared with circular pin fins. They observed that there was improvement of heat transfer and pressure loss characteristics for former fins. Analysis was performed for two different geometries of elliptical pin fins with different major axis and same minor axis length. Heat transfer coefficient for staggered configuration is calculated and compared with conventional circular fins [5].MonojBaruah, and P. Mahanta performed computational investigation over elliptical pin fins over rectangular tube arranged in staggered manner. They performed experimentation over solid elliptical pin fin and an elliptical pin fin with three elliptical perforations over the surface of the fins. Profiles for velocity, temperature and pressure for both profiles were determined and was observed that perforated fins show enhanced heat transfer [6].Subhash V. Jadhav, Prashant M. Pawar, Anil B. Shinde, Sandeep S. Wangikar, carried out numerical analysis on different geometries of pin fins heat sinks of micro channels. Three dimensional numerical analysis on elliptical pin fins using conjugate heat transfer module software was performed by

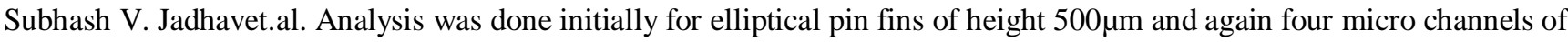
different fin layouts were analysed with various inclinations between major axis and fluid flow direction and for different axial distance between the fins. They have observed that variation of axial distance between the fins is better way to enhance heat transfer than that of former method [7].

\section{ASSUMPTIONS}

$>\quad$ Fluid in the pipe flows in one dimension.

Heat transfer in the pipe is one dimension only.

Flow in the pipe is turbulent with velocity $10 \mathrm{~m} / \mathrm{s}$. 
$>\quad$ All the fins are at the pipe base temperature.

Heat transfer from the fins is through conduction and natural convection

$>\quad$ Fluid flowing in the pipe is in single phase only.

Material of fins and pipe wall is same.

\section{DESCRIPTION}

A circular pipe which carries hot fluid with base temperature of $400 \mathrm{~K}$ is surrounded with number of circular annular fins and elliptical annular fins. Heat dissipation for a single elliptical annular fin is compared with the former one and the results were plotted using MATLAB results. Energy balance for the fin is made by balancing energy at any instant is equal to energy transfer to surroundings through conduction, convection and radiation. Since the fin base i.e., annulus of the fin is in direct contact of the wall of the pipe heat energy is transferred through conduction to the body of the fin and from the surface of the fin it is transferred through convection and radiation. Numerical modelling is done for both natural and forced convection. Temperature gradients for elliptical fins with different aspect ratios ranging from 0.1 to 0.9 were simulated and were compared to that of circular fins. Circular annular fins are designed with diameter of $0.2 \mathrm{~m}$ whose annulus is in direct contact with the wall of the pipe carrying hot fluid. Elliptical annular fins are designed with major axis length equal to the diameter of the fin but with different aspect ratios i.e., keeping the major axis constant minor axis is varied until aspect ratio 0.1 is reached. Numerical modelling is done for all the aspect ratio from 0.9 to 0.1 with step size of 0.1 but simulation is done for elliptical fins up to aspect ratio of 0.3. Results from numerical modelling are plotted for dimensionless temperature gradient with Fourier number. Fins were arranged around the circumference of the pipe at right angles without inclination i.e., the cross-sectional area of the fins is perpendicular to the cross-sectional area of the pipe. It is assumed that base temperature of all the fins is same. Since they are placed at $5 \mathrm{~mm}$ apart the temperature gradient between fin bases will be less. Transient analysis is performed for a time period of $100 \mathrm{sec}$. As the aspect ratio decreases the temperature lines on the fin surface are no more circular instead they get deviated from the centre of the fin. They show quadratic profile over the surface of the fins. As the aspect ratio increases the heat affected zone goes on decreasing. But whereas for elliptical annular fins with aspect ratios $0.9,0.8$ and 0.7 heat affected zone decreases when compared to circular annular fins. The geometry of the elliptical fins makes the external flow of fluid to be stream lined hence heat transfer can be more effective. As the aspect ratio is still reduced the temperature lines show hyperbolic nature i.e., they are curved away from fin centre.

\section{ENERGY BALANCE}

Energy available at any instant is transferred to atmosphere through convection, radiation and within the body through conduction.

$$
\begin{aligned}
& \frac{d}{d \tau}\left[m c_{p}\left(T_{i}-T\right)\right]=k A \frac{d t}{d x}+h_{\text {conv }}\left[T-T_{\infty}\right] A+\varepsilon c_{o} A\left[T^{4}-T_{\infty}^{4}\right] \\
& \frac{d}{d \tau}\left[\frac{4}{3} \pi a b l \rho c_{p}\left(T_{i}-T\right)\right]=k \frac{d t}{d x} \sqrt{2} \pi \sqrt{a^{2}+b^{2}}+h_{c o n v} \pi a b\left[T-T_{\infty}\right]+\varepsilon c_{o} \pi a b\left[T^{4}-T_{\infty}^{4}\right]
\end{aligned}
$$

Mass transfer balance over the surface of the fin

$$
\frac{d m}{d t}=h_{m}\left(\rho-\rho_{\infty}\right) A
$$


In non-dimensional form

$$
\frac{d T_{s}}{d x_{s}}=-\left(\frac{k_{2}(T s-1)+k_{3}\left(T s^{4}-1\right)}{\left(k_{4} x s-k_{1}\right)}\right)
$$

Variation of temperature with aspect ratio of the fin is as follows

$$
\frac{d T_{s}}{d n}=\left(\frac{c_{4}(T i-T s)-c_{2}(T s-1)-c_{2}\left(T s^{4}-1\right)}{\left(n c_{4}-c_{1}\right)}\right)
$$

In conventional process we consider all the heat conducted through the fin is dissipated to surroundings through convection. But in practice we encounter that even conduction is heat dissipating mode. It means that heat energy available at any instant in the fin is equal to heat energy dissipated though all the modes of heat transfer. Hence this problem is of mixed boundary condition. At the base of the fin energy available is equal to only conduction due to direct contact between base of the fin and wall. At the surface of the fin energy available is equal to conduction, convection and radiation. The effect of radiation is considered as the fluid (water) temperature is greater than its boiling point.

\section{RESULTS AND DISCUSSIONS}

Graphs for temperature gradient with time and surface temperature for both natural and forced convection of elliptical fins with different aspect ratios were plotted. It is observed that when these graphs are plotted using numerical modelling data slope of temperature gradient lines is increasing with aspect ratio. It results that as the aspect ratio of the fin increase temperature gradient increases. As the aspect ratio increases effectiveness of the fin also increases. The maximum efficiency of the fin increases with decrease in aspect ratio. This is due to increase of ratio of lateral surface area to cross-sectional area of the fin with reduction in aspect ratio. Variation of temperature gradient for elliptical fins in both natural and forced convection is numerically modelled and results were plotted. It is clearly observed that for natural convection as the aspect ratio increases the slope of the dimensionless temperature gradient with respect to surface temperature is increasing.

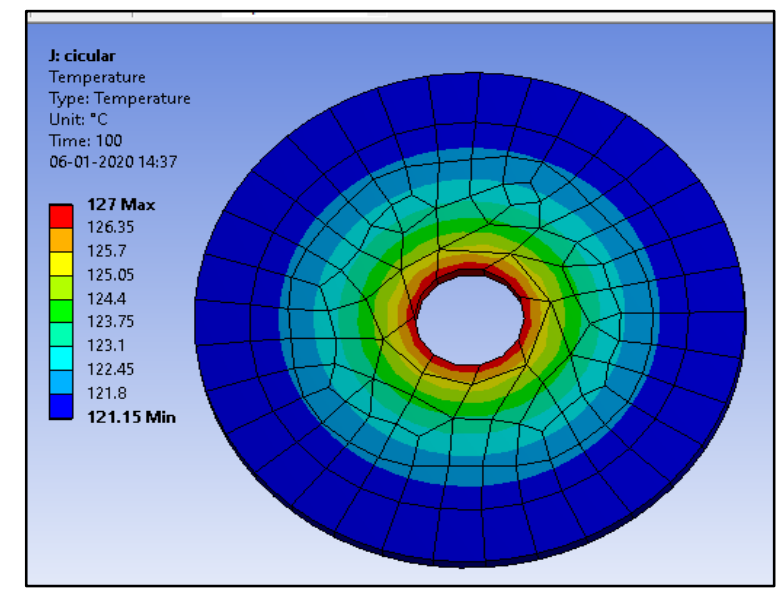

Figure 1: Temperature Profile of Circular Annular Fin 


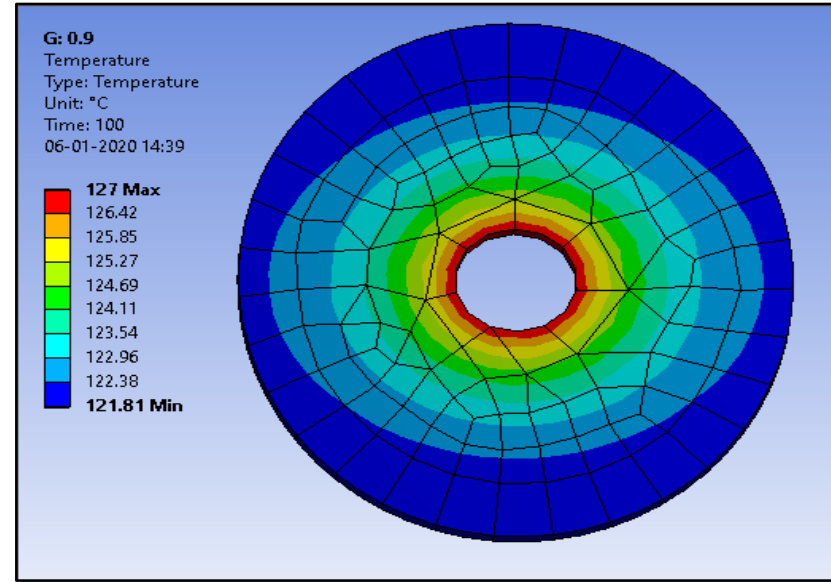

Figure 2: Temperature Profile for Elliptical fin with aspect Ratio 0.9

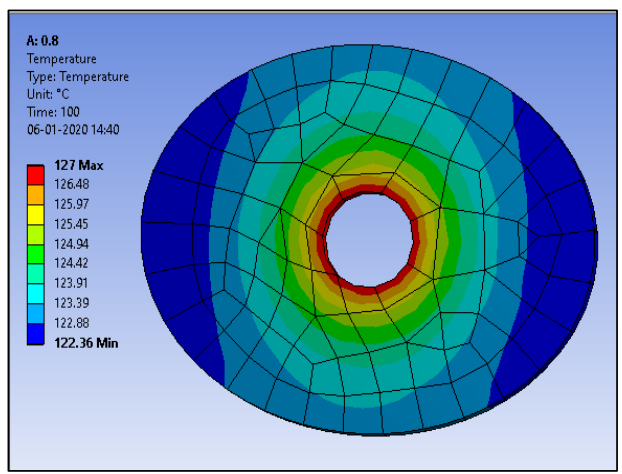

Figure 3: Temperature Profile for Elliptical fin with aspect Ratio 0.8

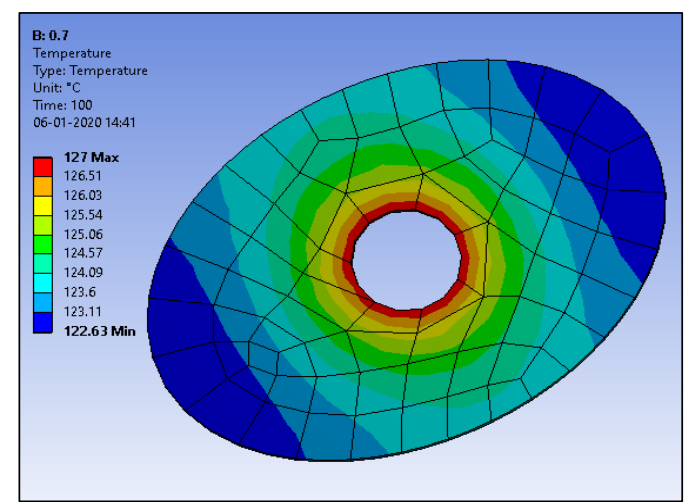

Figure 4: Temperature Profile for Elliptical Fin with aspect Ratio 0.7 


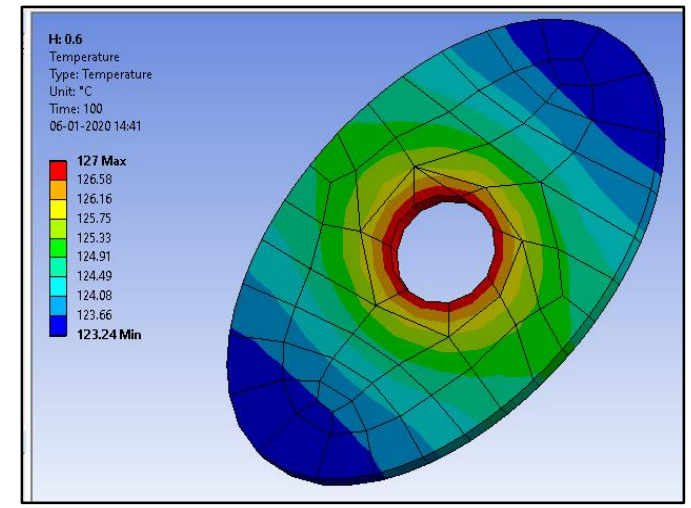

Figure 5: Temperature Profile for Elliptical Fin with Aspect Ratio 0.6

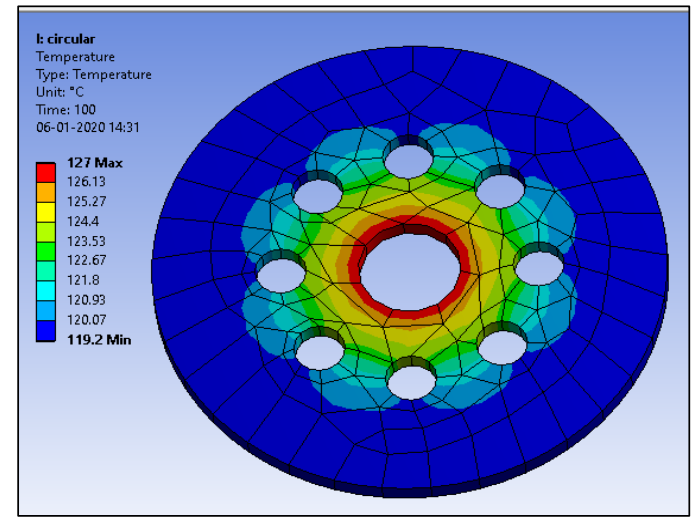

Figure 6: Temperature Profile for Circular Perforated Fin

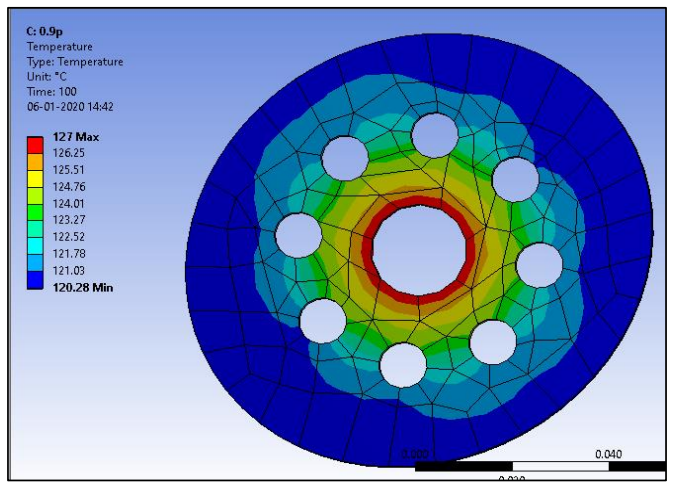

Figure 7: Temperature profile for perforated elliptical fin with aspect ratio 0.9

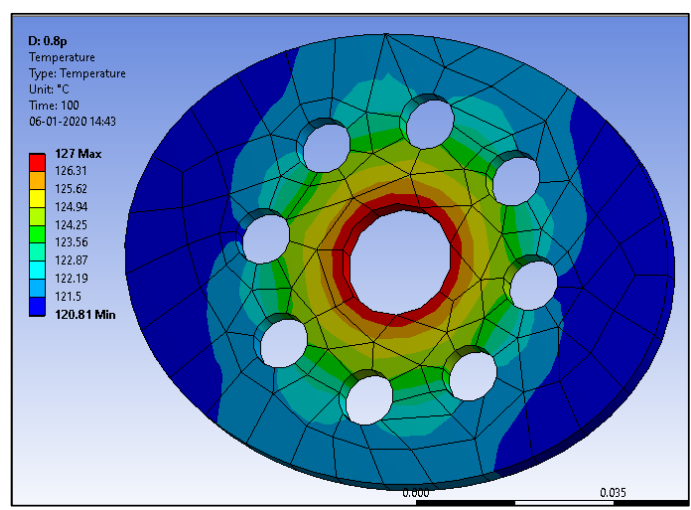

Figure 8: Temperature profile for perforated elliptical fin with aspect ratio 0.8 


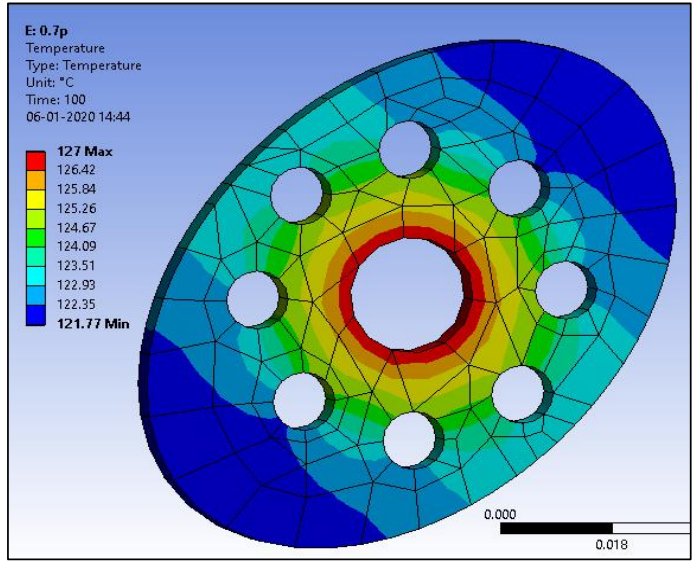

Figure 9: Temperature profile for perforated elliptical fin with aspect ratio 0.7

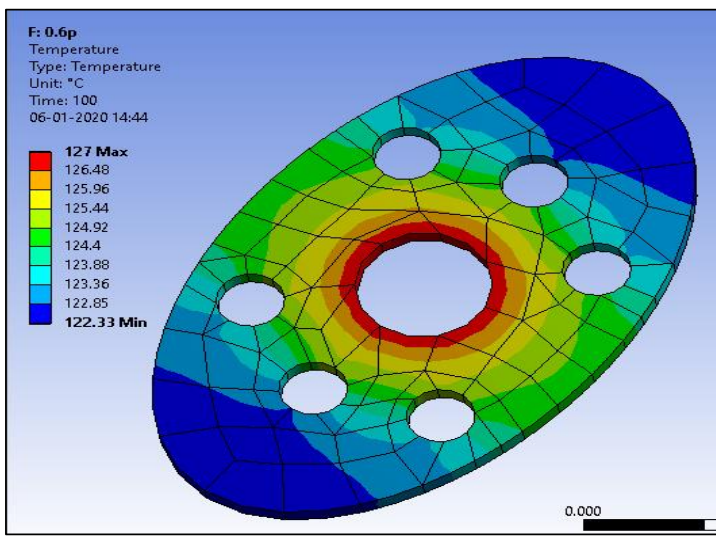

Figure 10: Temperature profile for perforated elliptical fin with aspect ratio 0.6.

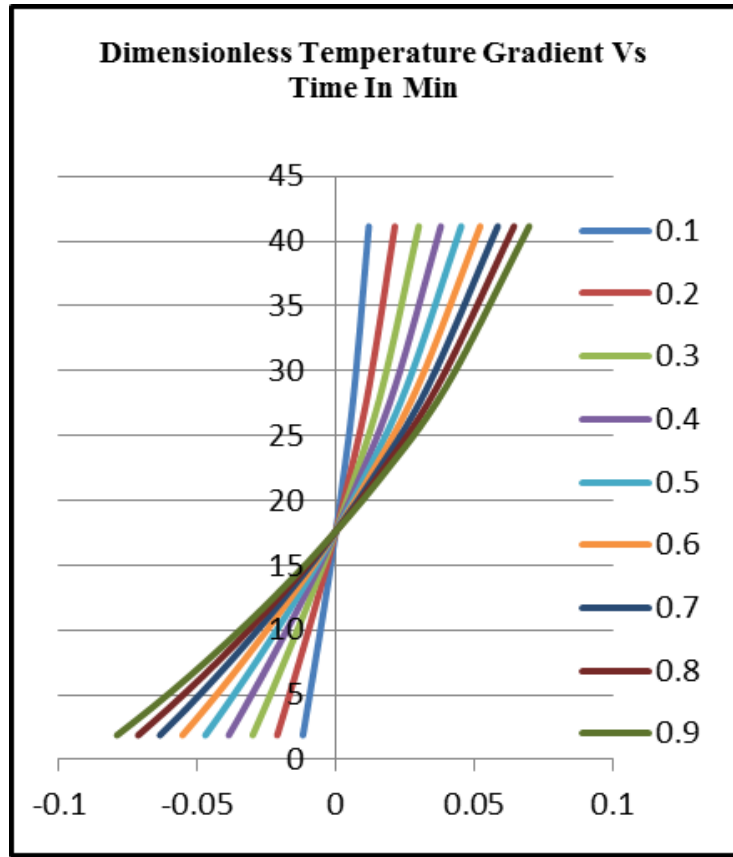

Figure 11: Variation of Temperature Dimensionless Temperature Gradient with Time 


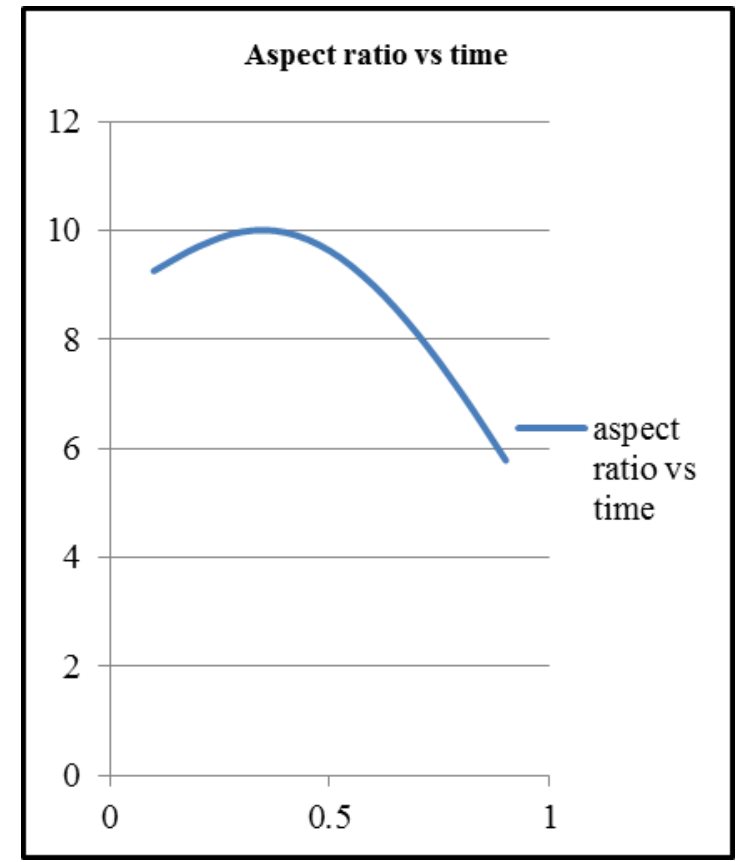

Figure 12: Variation of Aspect Ratio with Time for Fin

Table 1: Maximum and Minimum Temperatures Encountered by all Fins in Simulation

\begin{tabular}{|c|c|c|c|c|c|c|c|c|c|c|}
\hline \multirow[t]{2}{*}{ Temperature $\left({ }^{\circ} \mathrm{C}\right)$} & \multirow[b]{2}{*}{ circular } & \multirow[b]{2}{*}{$\begin{array}{l}\text { Circular } \\
\text { perforated }\end{array}$} & 0.9 & 0.8 & 0.7 & 0.6 & 0.9 & 0.8 & 0.7 & 0.6 \\
\hline & & & \multicolumn{4}{|c|}{$\begin{array}{l}\text { Elliptical fins of various aspect } \\
\text { Ratios }\end{array}$} & \multicolumn{4}{|c|}{$\begin{array}{l}\text { Elliptical perforated of various } \\
\text { aspect Ratios }\end{array}$} \\
\hline $\mathbf{M}$ & 12 & 12 & 127 & 127 & 127 & 127 & 127 & 127 & 127 & 127 \\
\hline Min & 121.15 & 119.2 & 121.81 & 122.36 & 122.63 & 123.24 & 120.28 & 120.81 & 121.77 & 122.33 \\
\hline
\end{tabular}

As the aspect ratio of the fins decreases from 1 i.e., circular fin to 0.1 the value of minimum temperature increases, and thermal zone is reduced which is clearly observed from the diagrams of simulation. Temperature gradient for circular fin and elliptical fins with aspect ratio 0.9, 0.8 and 0.7 are almost same. Material for elliptical fin manufacturing is less when compared to circular fins. For same temperature gradient the time taken for elliptical fin is less when compared with the former one. It is observed through simulation as the aspect ratio goes on decreasing heat affected zone in vertical direction is increasing and in the horizontal direction it is decreasing.

Obtained dimensionless temperature gradient in differential form is optimized using geometric programming. The optimal solution of the equation gives minimum temperature gradient for all the fins. The result was observed that temperature gradient for circular fin is minimum compared to rest of the fins.

Differential equation is reduced to geometric programming equation as follows:

Minimize $\mathrm{y}=2.5525 \mathrm{p}^{-1}+2.2192 \mathrm{p}^{-1} \mathrm{~T}_{\mathrm{s}}$

Subjected to $2.7878 \mathrm{p} \leq 1$

$$
0.75 \mathrm{~T}_{\mathrm{s}} \leq 1
$$

This is a one degree difficulty problem whose optimal value is 0.1325 at an aspect ratio of 0.925 . 


\section{FUTURE SCOPE}

Temperature gradient can be increased by using perforated fins which increases the rate of heat transfer through convection mode. Those results were shown numerically

\section{CONCLUSIONS}

- Temperature gradient for elliptical fins is more when compared with circular annular fins.

- When space is constrained for placing circular annular fins elliptical fins are suggestible.

- Heat affected zone for circular fins is more when compared to elliptical fins.

- Flow of air over elliptical fins is more streamlined when compared to circular fins.

- Elliptical fin has high stability and strength when compared to circular annular fins $\backslash$

- Variation of temperature profile is more streamlined than that of circular

- As the aspect ratio decreases from 1 to 0.7 effectiveness has observable progress.

- For manufacturing elliptical fin and circular fin whose major axis and diameter are same the former require less material and can fabricated in light weight.

\section{NOMENCLATURE}

$c_{p}$ Specific heat of hot fluid in the pipe $(\mathrm{kJ} / \mathrm{kg}-\mathrm{K})$

$T_{i}$ Initial temperature of the fin $(\mathrm{K})$

$\mathrm{T}$ Temperature of the fin at any instant $(\mathrm{K})$

$\mathrm{K}$ Thermal conductivity of the fin material (W/m-K)

A Cross sectional area of the fin $\left(\mathrm{m}^{2}\right)$

h Convective heat transfer coefficient of the fin $\left(\mathrm{W} / \mathrm{m}^{2}-\mathrm{K}\right)$

$\mathrm{T}_{\infty}$ Temperature of the fluid in the pipe $(\mathrm{K})$

$c_{o}$ stefan boltzmann constant $\left(\mathrm{W} / \mathrm{m}^{2}-\mathrm{K}^{4}\right)$

$\varepsilon$ emissivity of the fin material

$$
\begin{aligned}
c_{1}=\frac{2 a}{3 l}, c_{2}=\frac{2 h a^{2}}{3 l k}, c_{3} & =\frac{2 \varepsilon c T_{\infty}^{3} a^{2}}{3 l k}, c_{4}=\frac{2 a^{2} h_{m}\left(\rho-\rho_{\infty}\right)}{3 l \rho \alpha} \\
k_{1} & =\frac{3 l_{c}^{2}}{4 a b}, k_{2}=\frac{3 h l_{c}^{2}}{4 k l}, k_{3}=\frac{3 \varepsilon c T_{\infty}^{3} l_{c}^{2}}{4 k l}, k_{4}=\frac{3 l_{c}^{2} h_{m}\left(\rho-\rho_{\infty}\right)}{4 \alpha l \rho}
\end{aligned}
$$
A Major axis of the fin (m)
b Minor axis of the fin $(\mathrm{m})$
$\frac{d t}{d x}$ Temperature gradient of the fin along horizontal direction $\left({ }^{0} \mathrm{C} / \mathrm{m}\right)$
L Thickness of the fin (m)
$\rho$ Density of the hot fluid $\left(\mathrm{kg} / \mathrm{m}^{3}\right)$ 
$\eta$ Aspect ratio o the fin

$T_{s}$ dimensionless temperature

Fo Dimensionless time, Fourier number.

\section{REFERENCES}

1. N.Nagarani, Professor, Mechanical Engineering Department, Anna university of technology, Coimbatore, K.S.R College of Technology, Tiruchengode - 637 215, Tamilnadu, India, K.Mayilsamy, Head of the Department, Mechanical Engineering , Anna university of technology, Coimbatore Institute of Road and Transport Technology, Erode.Tamilnadu , India. "Experimental Heat Transfer Analysis on Annular Circular and Elliptical Fins", International Journal of Engineering Science and Technology Vol. 2(7), 2010, 2839-2845".

2. N.Nagarani, K.Mayilsamy, A. Murugesan," Fin Effectivenes optimization of elliptical Annular fins by Genetic Algorithm”, Sciverse Science Direct, Procedia Engineering, 38, 2012, 2939-2948.

3. H.Nemati, S.Samivand, "Performance optimization of annular elliptical fin based on thermo-geometric parameters", Volume 54, Issue 4, December 2015, Pages 1037-1042 Alexandria Engineering Journal [4] Hisham H. JasimEmailauthorMehmetSaitSöylemez," Optimization of a rectangular pin fin using elliptical perforations with different inclination angles", Journal of Mechanical Science and Technology October 2017, Volume 31, Issue 10, pp 5029-5039.

4. OguzUzol Pennsylvania State University, University Park, PA CengizCamci, Pennsylvania State University, University Park, PA, ” Elliptical Pin Fins as an Alternative to Circular Pin Fins for Gas Turbine Blade Cooling Applications: Part 1 “ Endwall Heat Transfer and Total Pressure Loss Characteristics", ASME Turbo Expo 2001: Power for Land, Sea, and Air June 4-7, 2001 New Orleans, Louisiana, USA.

5. MonojBaruah, and P. Mahanta, Department of Mechanical Engineering, Indian Institute of Technology Guwahati, Guwahati781039, INDIA, AnupamDewan, Department of Applied Mechanics, Indian Institute of Technology Delhi, New Delhi - 110016, INDIA, " Performance of Elliptical Pin Fin Heat Exchanger with Three Elliptical Perforations", CFD Letters, Volume -3(2), June 2011

6. Subhash V. Jadhav, Prashant M. Pawar, Anil B. Shinde, Sandeep S. Wangikar, SVERI's College of Engineering PandharpurIndia "Performance Analysis of Elliptical Pin Fins in the Microchannels", Techno-Societal 2018 pp 295-304, Springer Link.

7. B. Kundu, Department of Mechanical Engineering, Jadavpur University, Kolkata - 700 032, India, P.K. Das, Department of Mechanical Engineering, Indian Institute of Technology Kharagpur - 721 302, India, ” Performance analysis and optimization of elliptic fins circumscribing a circular tube" International Journal of Heat and Mass Transfer 50 (2007) 173-180, Science Direct 